\title{
Lumbar Spinal Stenosis: Pathophysiology and Treatment Principle: A Narrative Review
}

\author{
Byung Ho Lee, Seong-Hwan Moon, Kyung-Soo Suk, Hak-Sun Kim, Jae-Ho Yang, Hwan-Mo Lee \\ Department of Orthopedic Surgery, Yonsei University College of Medicine, Seoul, Korea
}

\begin{abstract}
Patients with lumbar spinal stenosis may exhibit symptoms such as back pain, radiating pain, and neurogenic claudication. Although long-term outcome of treatments manifests similar results for both nonsurgical and surgical treatments, positive effects such as short-term improvement in symptoms and decreased fall risk may be expected with surgery. Surgical treatment is basically decompression, and a combination of treatments can be added depending on the degree of decompression and the accompanying instability. Recently, minimally invasive surgery has been found to result in excellent outcomes in the treatment of lumbar spinal stenosis. Therefore, better treatment effects can be anticipated with an approach aimed at understanding the overall pathophysiology and treatment methods of lumbar spinal stenosis.
\end{abstract}

Keywords: Spinal stenosis; Pathophysiology; Diagnosis; Treatment; Surgery

\section{Introduction}

Degenerative lumbar spinal stenosis refers to the narrowing of the spinal canal due to degenerative changes in spinal joints, intervertebral discs, and ligamentum flavum [1]. As the space surrounding the neurovascular tissue becomes narrower, major clinical symptoms may appear, such as neurogenic claudication, radiating pain in the lower extremities, low back pain, and urinationdefecation impairment. Clinical symptoms may include decreasing sensation and fatigue in the lower extremities, as well as increasing pain in both buttocks or lower limbs, which may worsen when walking or standing for a long time (neurogenic intermittent claudication) [2]. Patients may experience alleviation of symptoms when they sit or bend forward [3], and if the pain caused by a walking or standing posture becomes severe, it may become difficult to perform daily and independent activities or the risk of fall may increase [3-5].

\section{Pathophysiology and Treatment Principle}

Degenerative lumbar spinal stenosis is a progressive disease that involves all the movement segments of the spine [6]. The relative instability initiated by degeneration of the intervertebral disc leads to hypermobility of the vertebral segments, resulting in increased pressure on the posterior facet joints, followed by a narrowing of the intervertebral disc space, an increased extension angle, and hypertrophy of the facet joints, especially the hypertrophy of the superior articular process. As it gradually progresses, the joints become ankylosed. The hypertrophic articular process causes localized joint stiffness (ankylosis). In addition, calcification or thickening of the yellow ligament is an important mech-

Received Sep 9, 2020; Revised Sep 10, 2020; Accepted Sep 11, 2020

Corresponding author: Hwan-Mo Lee

Department of Orthopedic Surgery, Yonsei University College of Medicine, 50-1 Yonsei-ro, Seodaemun-gu, Seoul 03722, Korea

Tel: +82-2-2228-2180, Fax: +82-2-363-1139, E-mail: hwanlee@yuhs.ac 
anism in stenosis. Joint tropism can also be a factor leading to degenerative lumbar spinal stenosis [7]. Consequently, this causes the stenosis of the spinal canal and compression of nerve structures, which may lead to intermittent neurogenic claudication due to congestion of the epidural venous blood and increased vascular pressure. Although minor trauma or repetitive stimulation associated with work does not significantly contribute to the occurrence of such stenosis, it can worsen the symptoms of existing stenosis. No symptoms can be felt when resting; however, symptoms appear due to the following reason when walking or standing. As the lumbar is extended, the spinal canal becomes narrower and the neural tissue is compressed, and simultaneously, the metabolic rate of nerve roots by the lower extremity movement increases, but the increase in blood flow cannot catch up with it. The cauda equina nerve roots gain metabolic energy from the blood circulation of the arteries located on the surface of nerve roots and the diffusion of cerebrospinal fluid. In lumbar spinal stenosis, when the nerve roots are under increased pressure in the spinal canal, neural ischemia and defective nerve conduction can occur. Symptoms can also occur due to venous congestion and may contribute to the etiology of lumbar spinal stenosis involving two or more segments. If the nerve roots are damaged, central sensitization of pain perception can occur, which can result in chronic pain.

\section{Anatomy}

Lumbar spinal stenosis is classified according to the anatomical location in which the spine is affected or to the etiology (Table 1). In degenerative lumbar spinal stenosis, the redundancy and loosening of yellow ligaments due to the narrowing of the disk space result in the narrowing of space in the spinal canal, which may be accompanied by instability. This relative hypermobility causes overgrowth and thickening of the posterior facet joints and surrounding ligaments. The yellow ligaments can thicken, especially in the area where they are attached to the spinal articular capsule around the lateral recess, which may compress the nerve roots (Fig. 1). These processes occur individually or simultaneously, resulting in symptoms of lumbar spinal stenosis.

Central stenosis refers to the narrowing of the space between both the posterior facet joints, primarily the space occupied by the dura sac and internal neural structures. The stenosis of this region is caused by the intervertebral
Table 1. Classification of spinal stenosis

\begin{tabular}{|c|c|}
\hline Variable & Classification \\
\hline \multicolumn{2}{|l|}{ Anatomic } \\
\hline Anatomic area & Anatomic region (local segment) \\
\hline \multirow[t]{2}{*}{ Cervical } & Central \\
\hline & Foraminal \\
\hline \multirow[t]{4}{*}{ Thoracic } & Central \\
\hline & Lateral recess \\
\hline & Foraminal \\
\hline & Extraforaminal (far-out) \\
\hline \multicolumn{2}{|l|}{ Pathologic } \\
\hline \multirow[t]{4}{*}{ Congenital } & Achondroplastic (dwarfism) \\
\hline & Congenital forms of spondylolisthesis \\
\hline & Scoliosis \\
\hline & Kyphosis \\
\hline Idiopathic & - \\
\hline \multirow[t]{6}{*}{ Degenerative and inflammatory } & Osteoarthritis \\
\hline & Inflammatory arthritis \\
\hline & Diffuse Idiopathic skeletal hyperostosis \\
\hline & Scoliosis \\
\hline & Kyphosis \\
\hline & Degenerative forms of spondylolisthesis \\
\hline \multirow[t]{2}{*}{ Metabolic } & Paget disease \\
\hline & Fluorosis \\
\hline
\end{tabular}

disc extrusion, bulging of the annulus fibrosis, osteophyte formation, and folded or thickened yellow ligaments. Symptomatic central stenosis results in neurogenic claudication with pain in the lower extremities. The lateral side of the dural sac is known as the lateral canal, through which nerve roots pass, and when the nerve roots are compressed, there might be radiating pain.

The lateral recess is known as the "entrance zone," which begins at the medial border of the superior articular process and extends to the medial border of the pedicle [6]. In this region, the nerve roots that have emerged from the dura sac pass through the distal and lateral parts. The boundaries of the lateral recess comprise a vertebral pedicle on the lateral side, a superior articular process of the posterior joint on the dorsal side, an intervertebral disc and posterior ligaments on the anterior side, and a spinal canal on the medial side. The primary causes of stenosis in the entrance zone are a posteriorly herniated intervertebral disc and hypertrophic osteoarthritis of the 
posterior joints, wherein the nerve roots are compressed by the thickened superior articular process.

The mid-zone refers to the foraminal region located in front of the pars [8]. The anatomical structure corresponds to the ventral part of the pars. Regarding the boundary structures, they medially border on the lateral recess, anteriorly on the vertebral body and intervertebral disc, posteriorly on the pars and intertransverse ligaments, and laterally on the surface of the pedicle. Stenosis occurs in this region following the thickening of the fibrocartilage tissue at defective pars or by compression due to osteophytes. The thickening of the yellow ligament sometimes causes stenosis up to the neural foramen, and the formation of osteophytes under the pars defect is also related to stenosis occurring in this region (Fig. 2). In spondylolisthesis and degenerative scoliosis, one vertebral pedicle is relatively lowered due to the rotational malalignment of the vertebral body or asymmetrical narrowing of the intervertebral disc. This can also cause neural compression at this level.

The exit zone is shaped as an inverted teardrop created by the intervertebral foramen, and the neural foramen is
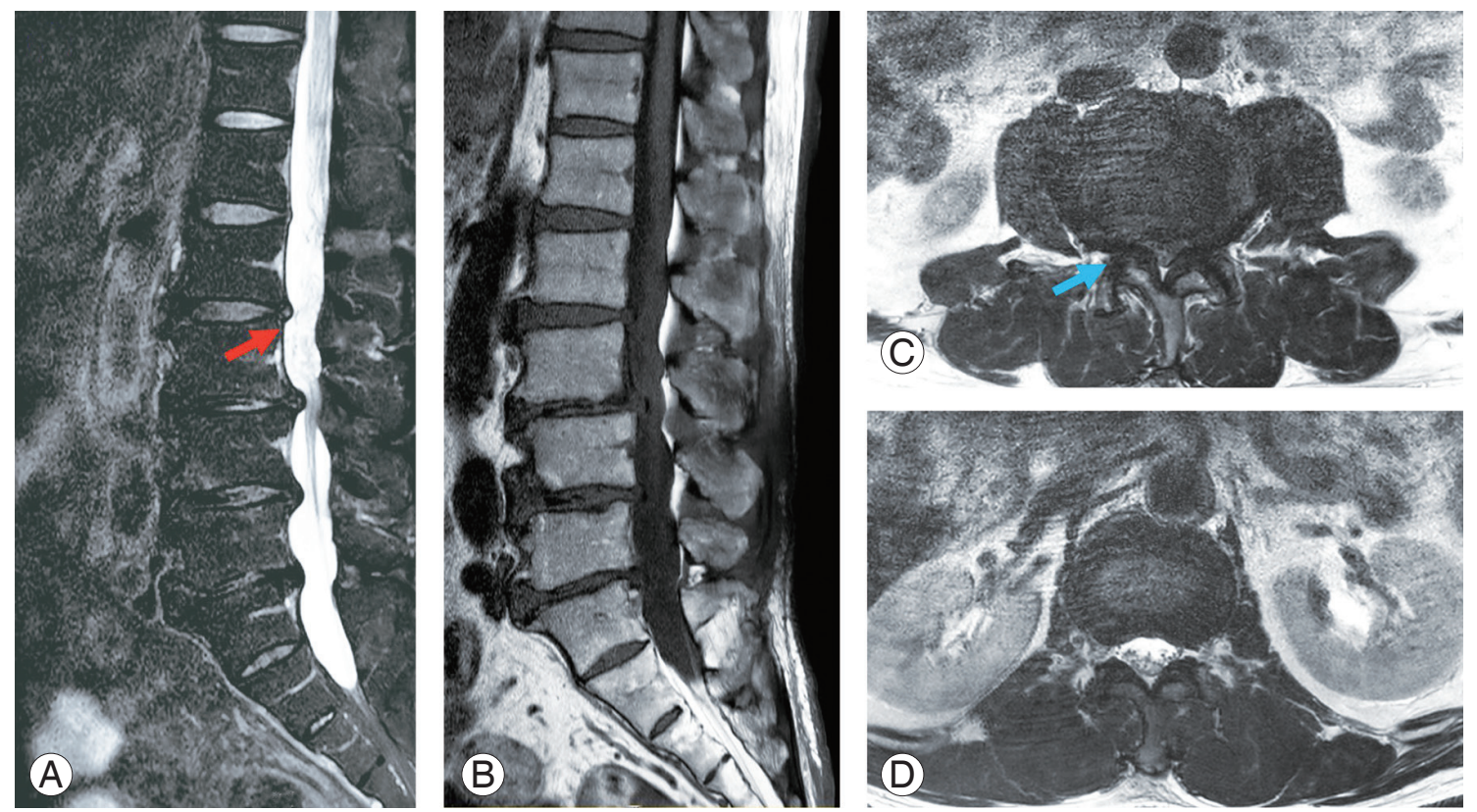

Fig. 1. Magnetic resonance imaging image in patients with spinal stenosis. (A, B) In the sagittal image scan, central stenosis and resultant crowding of cauda equina are seen (arrow in red). (C, D) In the axial scan, the stenosis segment can be easily compared with the normal segment (arrow in blue).
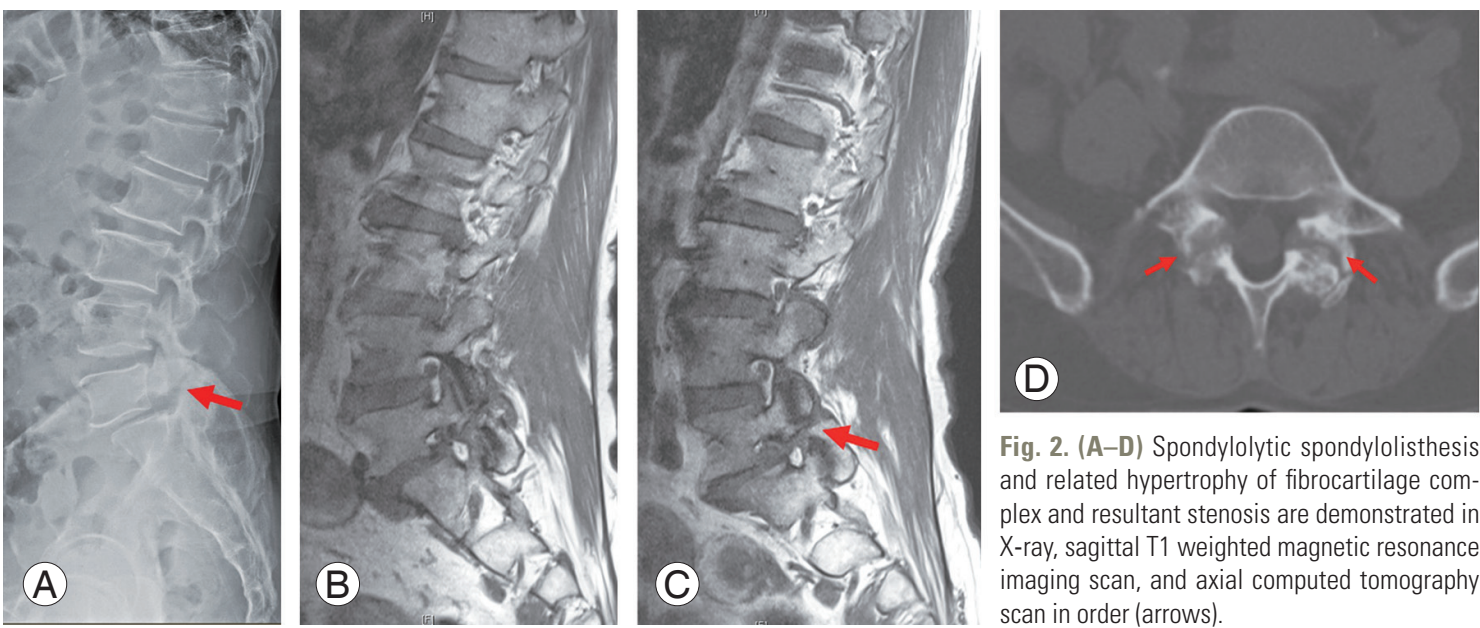

Fig. 2. (A-D) Spondylolytic spondylolisthesis and related hypertrophy of fibrocartilage complex and resultant stenosis are demonstrated in $\mathrm{X}$-ray, sagittal $\mathrm{T} 1$ weighted magnetic resonance imaging scan, and axial computed tomography scan in order (arrows). 
the space between the lower part of the upper pedicle and the upper part of the pedicle of the lower vertebrae, which borders posteriorly on the pars, inferior articular process of the upper vertebrae, and the superior articular process of the lower spine and anteriorly on the posterior surface of the vertebral body and the intervertebral disc [6]. The dorsal root ganglions and ventral motor roots occupy approximately $30 \%$ of this space. This is the region that changes into the epineurium, which wraps the nerve roots in the dura. Nerve roots are located in this region and can be compressed by the protrusion of the far lateral disk, subluxation related to spondylolisthesis, or arthritis [9]. The most common cause is degenerative spinal arthritis.

Degenerative lumbar spinal stenosis primarily proceeds due to the degenerative process of the vertebral joint and yellow ligament [10], and imaging studies can reveal progressed spondyloarthrosis. These abnormal findings can be found primarily on both sides, and the most common regions are between L4 and L5, followed by between L5 and sacrum and between L3 and L4 [11]. In addition, disk herniation and spondylolisthesis can exacerbate acutely such stenosis-related clinical symptom. Spondylolisthesis and degenerative spondyloarthropathy rarely cause stenosis in young patients, but when these degenerative changes occur with aging, spondylolisthesis, and spondyloarthropathy in patients aged $>50$ years, they can result in lateral recess or foraminal stenosis.

\section{Natural History}

Lumbar spinal stenosis is often asymptomatic. Studies have reported no close association between clinical symptoms and anteroposterior diameter of the spinal canal. The natural course of lumbar spinal stenosis in the majority of cases includes gradual development of symptoms; however, in some cases, it becomes acute following some trauma or severe physical activity. In several patients, even when imaging tests lead to the diagnosis of stenosis, symptoms and physical examination may only reveal negligent abnormalities. Several studies have reported that patients who received surgical treatment, such as decompression, exhibit better progress than those who received nonsurgical treatment; however, $50 \%$ of the patients who received nonsurgical treatment and were followed up for 8-10 years showed improvements in back pain and leg pain. In a prospective randomized study involving 100 patients with symptoms of stenosis who were provided surgical or nonsurgical treatment, it was observed that symptoms improved after approximately 3 months irrespective of the type of treatment, and some patients showed symptom improvement after 12 months. In the nonsurgical treatment group, symptoms worsened over time, but after 4 years, approximately $50 \%$ of the patients displayed excellent or fair progress. In contrast, $80 \%$ of the patients who received surgical treatment exhibited good results at the 4-year follow-up [12]. In another study, the majority of patients diagnosed with lumbar spinal stenosis exhibited stable disease progression, and $15 \%-50 \%$ of those who received nonsurgical treatment showed good results. Worsening of symptoms even with appropriate conservative treatment may be an indication for surgical treatment. According to Weinstein et al. [12], the surgical treatment group showed improvement in all primary outcomes compared with the nonsurgical treatment group.

\section{Prevalence}

The overall prevalence of lumbar spinal stenosis varies among studies. The prevalence in the group based on clinical diagnostic criteria has been reported to be approximately $11 \%$ in the general population [13-15]. According to the diagnosis based on imaging studies, the prevalence was found to be $11 \%$ in the asymptomatic general population and $38 \%$ in the general population. Prevalence increases with age, and the diagnosis based on imaging tests such as magnetic resonance imaging (MRI) and computed tomography (CT) scan is known to be made a decade earlier than the diagnosis based on clinical symptoms. In some studies in which the International Classification of Diseases codes were used, the prevalence was reported as $7 \%-23 \%$. Some expert opinions reported a prevalence of $4 \%-53 \%$. Considering the progression of this degenerative disease, the prevalence of degenerative lumbar spinal stenosis can be significantly influenced by age. For instance, one study reported a prevalence of approximately $78 \%$ in the target patients with an average age of 67 years (age range, 40-93 years), whereas the prevalence was only approximately $12 \%$ in patients with an average age of 40 years [8]. Some prevalence studies used only central stenosis as the diagnostic criterion. In other studies, both foraminal and lateral access stenosis were included. Moreover, a bias may be introduced due to the average age of the target patient group. Therefore, it is important to consider these aspects when interpret- 
ing the results of the abovementioned previous studies $[3,9,10,16,17]$. Previously, lumbar spinal stenosis has been reported to be more common in men; however, recent reports have mentioned that it is $3-5$ times more common in women $[11,18]$.

\section{Clinical Evaluation}

Till date, there exists some ambiguity in the clinical diagnostic criteria for degenerative lumbar spinal stenosis. According to a study conducted in 2016, the consensus of experts on clinical symptoms included (1) pain in the lower extremities or buttocks while walking, (2) symptom relief resulting from bending the lower back forward, (3) symptom relief from bending the back forward while using a shopping cart or riding a bicycle, (4) motor or sensory disturbance while walking, (5) normal and symmetrical pulse of the dorsalis pedis artery, (6) weakening of lower extremity, and (7) lower back pain $[13,14]$. In other studies, detailed history, gait, and some physical examination findings were also included in the classification criteria $[13,15,19]$.

Patients with lumbar spinal stenosis generally test negative in the straight leg raising test, and findings of motor neurological disorders are rare. Sudden appearance or exacerbation of sciatic neuralgia suggests the possibility of occurrence of intervertebral disc herniation in addition to existing spinal stenosis. Radiating pain occurs often in the lateral access and foraminal stenosis, and L5 neurological symptoms are the most common [20]. In neurological examination, motor deficit can be primarily observed due to the compression of L5 nerve roots in severe stenosis or degenerative spondylolisthesis. In the event of paresthesia, peripheral neuropathy caused by diabetes, alcoholism, or drug use should be suspected. For detecting the interruption in blood circulation, the dorsalis pedis and popliteal arteries should be palpated, and the edema and varicose veins in lower extremities should be excluded [21]. Moreover, it must be differentiated from the symptoms of lesions in the lower limbs, such as hip joints and knees [22].

\section{Diagnostic Imaging}

\section{Radiography}

Although establishing the diagnosis of stenosis using a simple radiographic examination is not confirmative, the following factors may help diagnose the stenosis: a short vertebral pedicle shown in the lateral image, a narrow interpedicle distance shown in the anteroposterior image, calcification of the ligaments or intervertebral discs, narrowing of the foramen, and hypertrophy of the posterior joints. It is possible to determine whether fusion is required by checking the presence of segmental instability shown in the flexion-extension lateral image. A translation of $>4-5 \mathrm{~mm}$ or changes in angular motion of $>10^{\circ}-15^{\circ}$ indicate segmental instability $[11,23]$.

\section{Magnetic resonance imaging}

MRI is useful for diagnosing lumbar spinal stenosis and identifying the degree of degenerative change and the size of the spinal canal $[1,24,25]$. A low correlation exists between the degree of morphological stenosis observed in MRI and clinical symptoms. In other words, the degree of stenosis observed in MRI and the severity of clinical symptoms are not proportional [26]. MRI may be used to confirm diagnosis in patients with persistent neurological claudication or radiating pain, but it should not be used for screening purposes as a high proportion of patients exhibit no symptoms even when the stenosis is confirmed in MRI. In sagittal T2-weighted images, a myelogramlike image is observed, whereas in sagittal T1-weighted images, foraminal stenosis can be well identified. In case normally distributed fat tissue is not detected around the nerve roots, foraminal stenosis can be diagnosed (Fig. 3). In the case of axial cut images, central stenosis and internal neural structures can be clearly confirmed in T1- and T1-weighted images, respectively. Far lateral disk protrusion can be confirmed when there is a loss of fat tissue normally distributed between the intervertebral disc and nerve roots shown on axial cut T1-weighted images. However, when spinal deformities, such as scoliosis, are accompanied, spondylolisthesis is severe, or when they extend beyond the scan range of sagittal and axial planes, obtaining scan images of the desired parts may be difficult, which results in inaccurate information (Fig. 4). Recently, intraspinal diffusion tensor imaging parameters such as apparent diffusion coefficient and fractional anisotropy have been reported to be useful for the quantitative assessment of lumbar spinal stenosis [27]. 

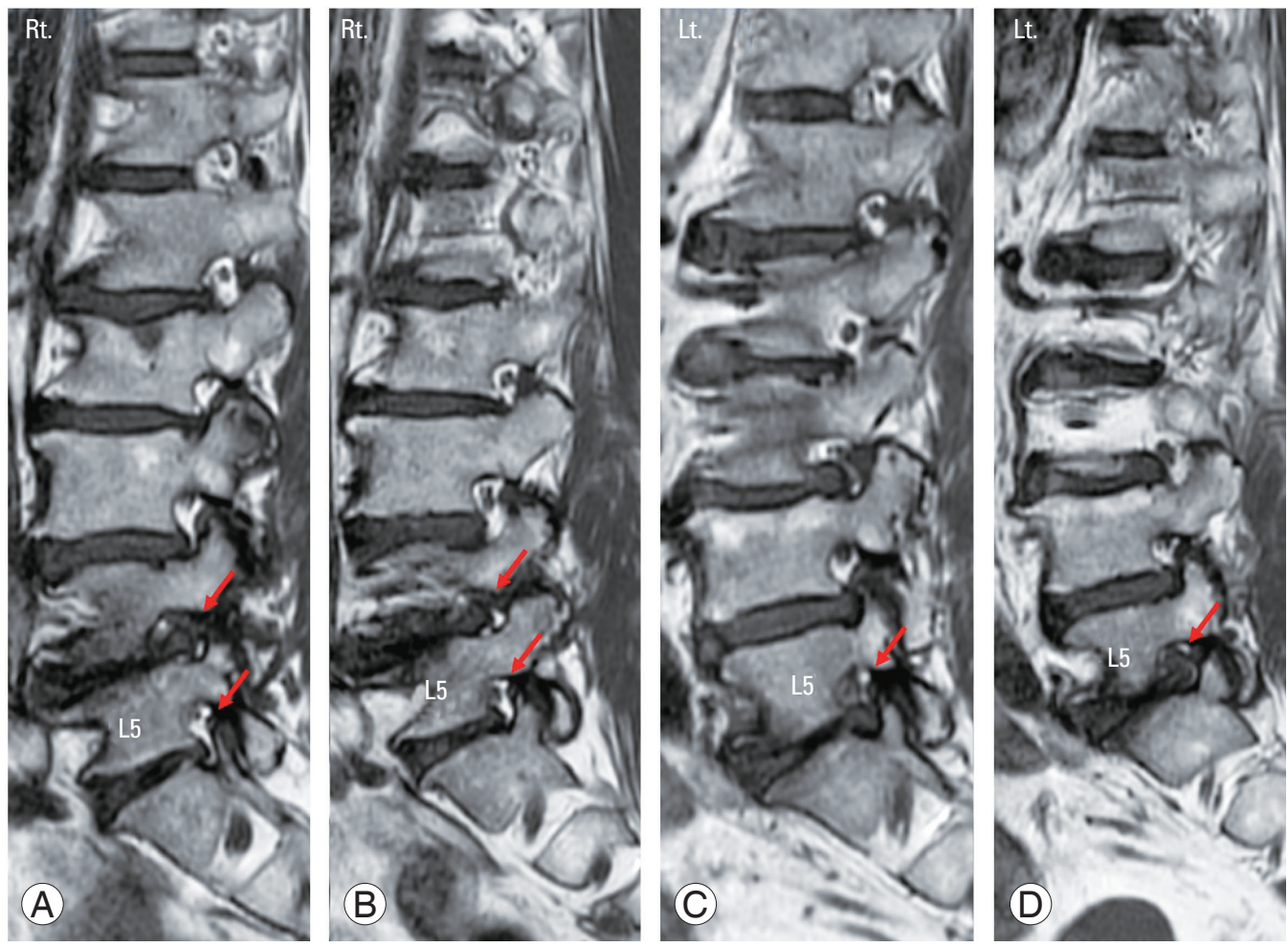

Fig. 3. (A-D) In a sagittal T1 weighted magnetic resonance imaging scan, foraminal stenoses are well visualized with loss of perineural fat tissue compared with normal foramen (arrows in red). Rt., right; Lt., left.
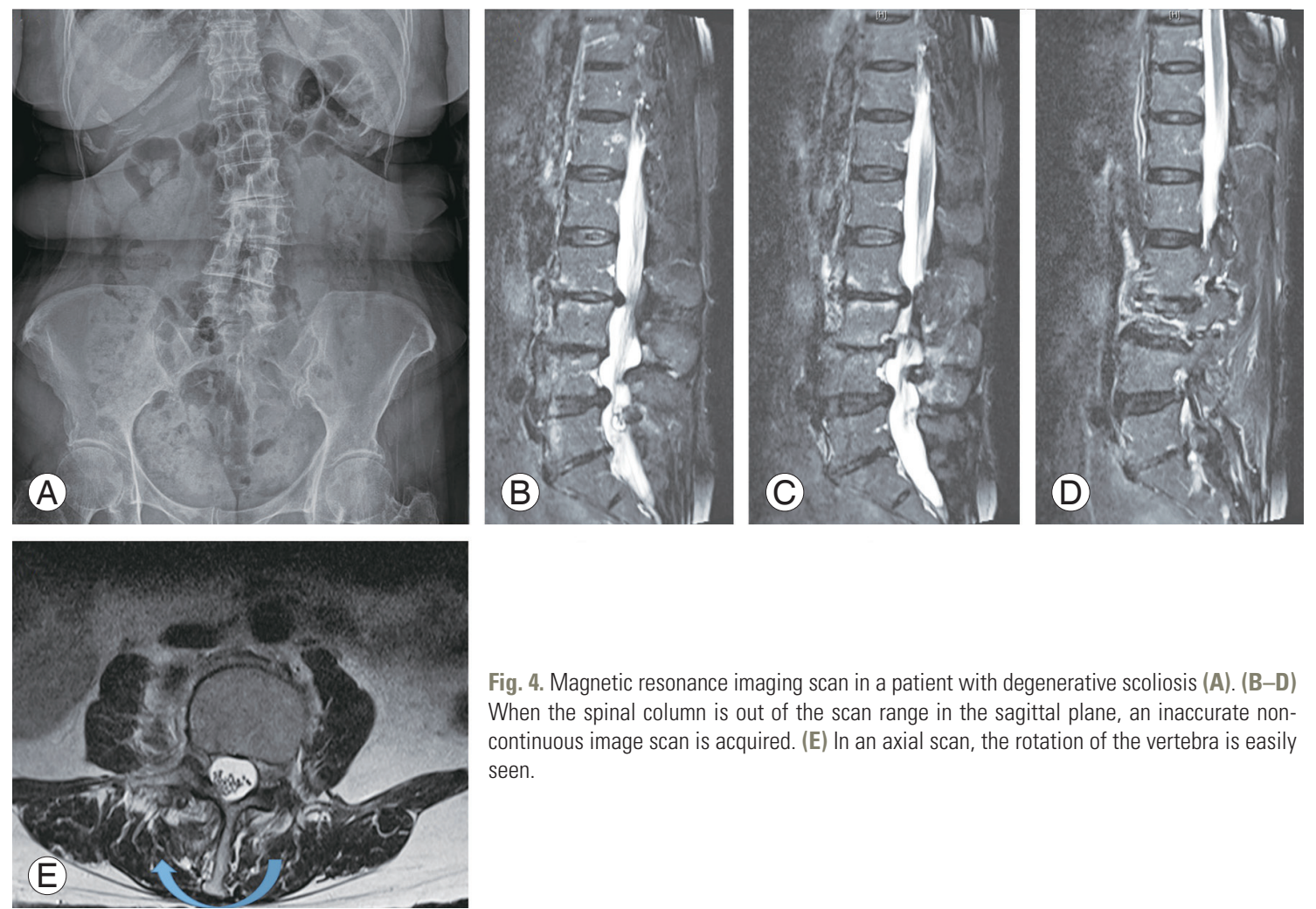

Fig. 4. Magnetic resonance imaging scan in a patient with degenerative scoliosis (A). (B-D) When the spinal column is out of the scan range in the sagittal plane, an inaccurate noncontinuous image scan is acquired. (E) In an axial scan, the rotation of the vertebra is easily seen. 


\section{Computed tomographic myelography}

CT is also an imaging test that is often used in surgery planning in patients with stenosis [28]. CT, after myelogram, provides more accurate information. Myelography, followed by CT, is the best suited approach for patients with dynamic stenosis, postoperative leg pain, severe scoliosis or spondylolisthesis, metallic implants, contraindications to MRI, and lower extremity symptoms in the absence of findings on MRI. However, as myelogram is invasive and can be accompanied by side effects such as headache, nausea, and seizures, the frequency of its use has decreased in recent years. Using myelography, dynamic images can be obtained through lateral images, while flexion and extension are performed in the standing position, which are not available in the case of MRI examination.

In addition, as $\mathrm{CT}$ provides more information regarding the bone anatomy, such as calcification of bone spurs or intervertebral discs, than MRI, information that is essential for decompression surgery may actually be obtained [29]. Moreover, CT is a better alternative imaging method for patients with cardiac pacemakers who cannot undergo MRI for diagnostic purposes.

\section{Other diagnostic studies}

Electromyography can be conducted when the diagnosis of neuropathy is uncertain in patients with comorbidities such as diabetes [30]. Furthermore, the vascular Doppler test can be used to rule out lesions caused by vascular problems in the lower extremities [21]. One study reported that a bicycle-Bruce protocol can contribute to the diagnosis [31].

\section{Nonoperative Treatment}

Nonsurgical treatment is appropriate for patients with mild to moderate symptoms. Commonly used treatment strategies include bed rest for a short period of approximately 1 week, medication (anti-inflammatory drugs, oral adrenal corticosteroids, muscle relaxants, prostaglandin E1 analogs, antidepressants, and anticonvulsants such as gabapentin), lumbar exercise (isometric flexion exercise and hyperextension exercise), physical therapy, orthosis usage, thermal therapy, ultrasound, massage, electrical stimulation, and traction therapy [21,32].

\section{Epidural steroid injection}

Epidural steroid injection is an intermediate step between conservative and surgical treatments. Spinal stenosis can result in nerve edema due to structural and chemical stimulation of the nerve roots caused by physical compression of the nerve tissue and nerve root inflammation due to local ischemia caused by congestion of venous blood around the nerve roots, which may lead to the release of phospholipase or leukotriene $\mathrm{B}$, worsening inflammatory reactions, and edema. The purpose of epidural steroid injections for stenosis is to induce a strong anti-inflammatory action to reduce the inflammatory response and edema through the reduction of leukocyte migration, inhibition of cytokine production and release, and cell membrane stabilization. In several studies, epidural steroid injection treatment was found to produce a short-term relief of symptoms in approximately $50 \%-87 \%$ of the cases $[26,33$ 35]. Indications for epidural steroid injections include acute radiating pain and neurological claudication that interfere with daily life, despite the administration of pain relievers and rest, which are anticipated to improve the symptoms. There are also reports of recent studies using epidural neuroplasty and other combination of drugs such as ropivacaine and dexmedetomidine related to thoracolumbar surgery $[29,30]$.

\section{Principles of spinal stenosis surgery}

The decision to perform surgery is based on the complaints of loss of ability in daily life, such as limited walking due to pain, weakening of the muscles, or paresthesia in the buttocks or lower extremities after adequate conservative treatments for at least 2-3 months.

Surgeries are rarely performed for lower back pain that is caused only by spondylolisthesis and scoliosis without instability. Although rare, even when long-lasting motor nerve palsy is the only symptom, other causes must be identified before performing surgery because of the difficulty of predicting the likelihood of recovery after surgery. In case there exists a relatively rapidly progressing nerve impairment or loss of urination-defecation functions, early decompression is required [36]. When deciding on surgery, abnormal findings from CT or MRI imaging should match the patient's symptoms [16].

The principle of surgical treatment is sufficient decompression of nervous structures [37]. When sufficient 
decompression is accompanied by instability due to the removal of excessive bone structures, isthmic or degenerative spondylolisthesis, scoliosis, or kyphosis, a combination of fusion surgery should be considered (Figs. 5, 6). Other criteria for fusion include adjacent segmental degeneration due to a previously performed fusion, recurring stenosis, or intervertebral disc herniation after a previous surgery. Laminectomy alone could be considered when severe multisegment stenosis is present in elderly patients. During decompression, less than half the medial part of each posterior joint of one spinal segment is excised to obtain sufficient decompression in the center and outside without causing instability to the segments. More localized decompression is possible when the exact symptom-causing sites are identified through a selective nerve root block.

When decompression is performed, care should be taken to reduce the risk of dural damage by checking for adhesion of the neural membrane that may exist even without a history of surgery. When the stenosis of the lateral recess and foramen is very severe, caution is required during decompression as it may result in neural damage with surgical devices.

When fusion is performed, it is important to consider sagittal balance as achieving sagittal balance after surgery can result in good surgical outcomes and also help reduce the risk of fall after surgery [38,39]. Studies have reported that the recent emergence of surgical techniques using minimally invasive spine surgery, including endoscopic decompression, has led to better results in terms of length of hospital stay, blood loss, cost, and postoperative outcome than the existing surgical methods [36,40-43].

\section{Conclusions}

Patients with lumbar spinal stenosis may exhibit symptoms such as back pain, radiating pain, and neurogenic claudication. Although long-term outcome of treatments manifests similar results for both nonsurgical and surgical treatments, positive effects such as short-term improvement in symptoms and decreased fall risk may be expected with surgery. Surgical treatment is basically decom-
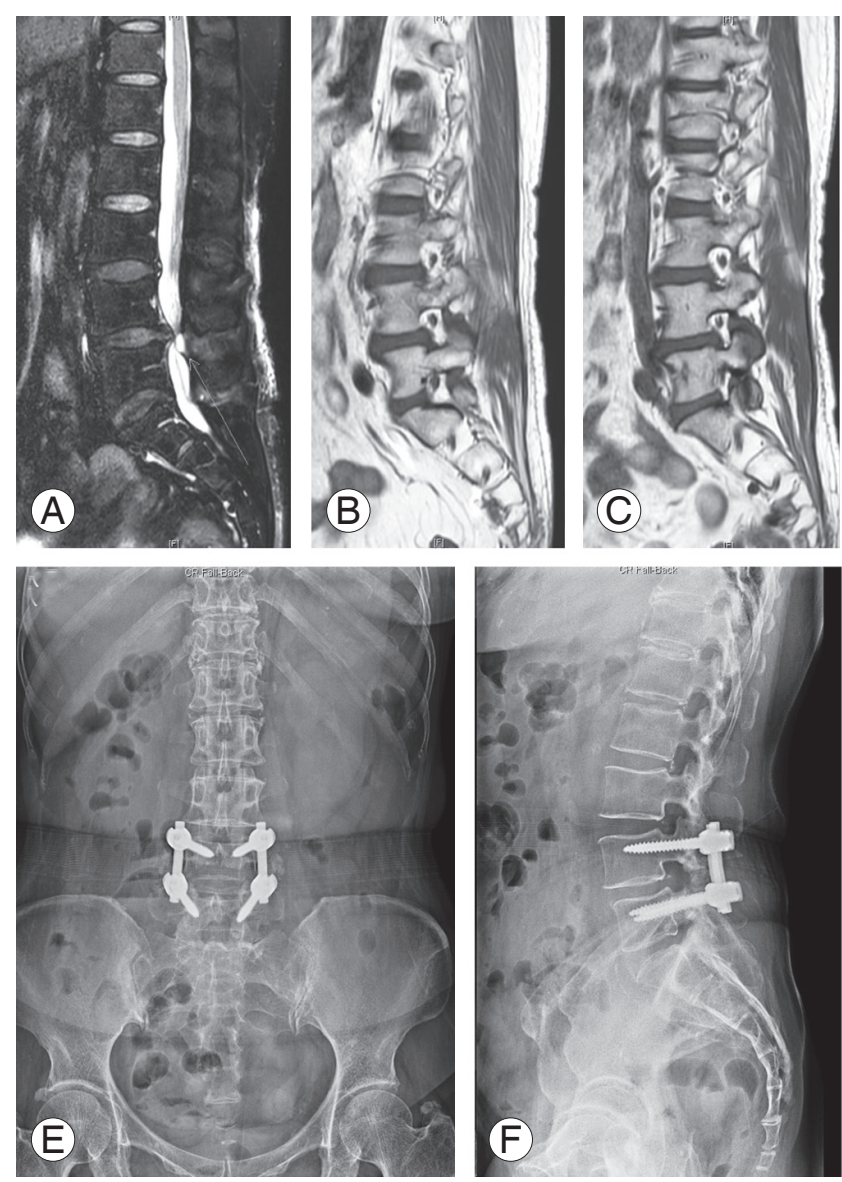

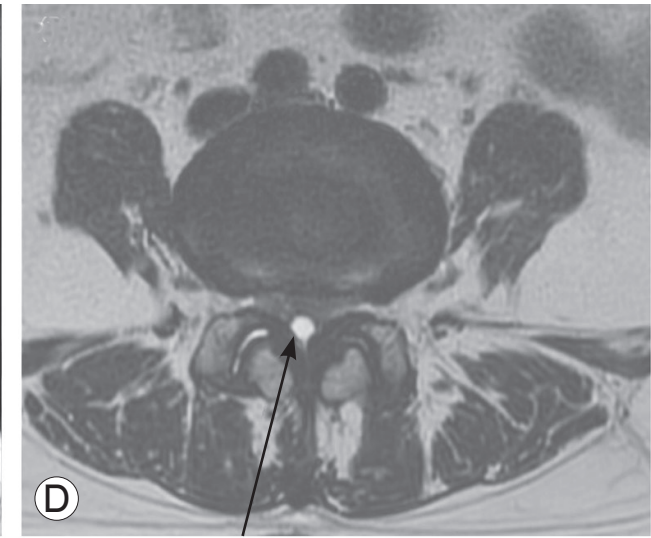

Fig. 5. Sixty-one-year-old female patient with central stenosis. (A-D) On magnetic resonance imaging sagittal and axial scan, central stenosis with cyst in ligamentum flavum and facet arthrosis are seen (arrow). (E, F) Decompression and posterolateral fusion with instrumentation between $\mathrm{L} 4-5$ were done. 

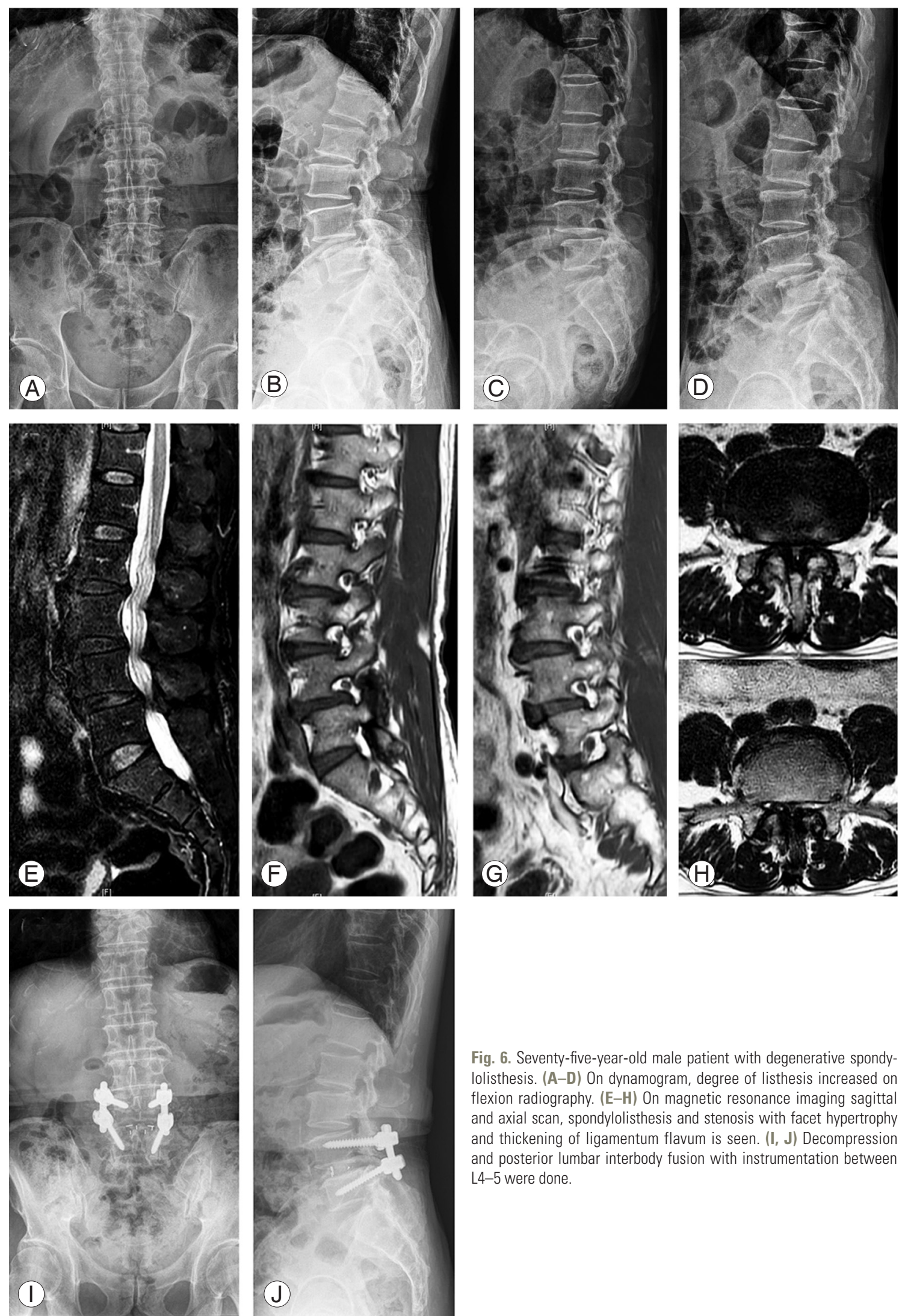

Fig. 6. Seventy-five-year-old male patient with degenerative spondyIolisthesis. (A-D) On dynamogram, degree of listhesis increased on flexion radiography. (E-H) On magnetic resonance imaging sagittal and axial scan, spondylolisthesis and stenosis with facet hypertrophy and thickening of ligamentum flavum is seen. $(\mathbf{I}, \mathbf{J})$ Decompression and posterior lumbar interbody fusion with instrumentation between L4-5 were done. 
pression, and a combination of treatments can be added, depending on the degree of decompression and the accompanying instability. Recently, minimally invasive surgery has been reported to be excellent in the treatment of lumbar spinal stenosis. Therefore, better treatment effects can be expected with an approach aimed at understanding the overall pathophysiology and treatment methods of lumbar spinal stenosis.

\section{Conflict of Interest}

No potential conflict of interest relevant to this article was reported.

\section{References}

1. Katz JN, Harris MB. Clinical practice: lumbar spinal stenosis. N Engl J Med 2008;358:818-25.

2. Rauschning W. Normal and pathologic anatomy of the lumbar root canals. Spine (Phila Pa 1976) 1987;12:1008-19.

3. Iversen MD, Katz JN. Examination findings and selfreported walking capacity in patients with lumbar spinal stenosis. Phys Ther 2001;81:1296-306.

4. Lee BH, Kim TH, Park MS, et al. Comparison of effects of nonoperative treatment and decompression surgery on risk of patients with lumbar spinal stenosis falling: evaluation with functional mobility tests. J Bone Joint Surg Am 2014;96:e110.

5. Kim HJ, Chun HJ, Han CD, et al. The risk assessment of a fall in patients with lumbar spinal stenosis. Spine (Phila Pa 1976) 2011;36:E588-92.

6. Lee CK, Rauschning W, Glenn W. Lateral lumbar spinal canal stenosis: classification, pathologic anatomy and surgical decompression. Spine (Phila Pa 1976) 1988;13:313-20.

7. Abbas J, Peled N, Hershkovitz I, Hamoud K. Facet tropism and orientation: risk factors for degenerative lumbar spinal stenosis. Biomed Res Int 2020;2020:2453503.

8. Kjaer P, Leboeuf-Yde C, Korsholm L, Sorensen JS, Bendix T. Magnetic resonance imaging and low back pain in adults: a diagnostic imaging study of 40-year-old men and women. Spine (Phila Pa 1976) 2005;30:1173-80.

9. Jensen RK, Jensen TS, Koes B, Hartvigsen J. Prevalence of lumbar spinal stenosis in general and clinical populations: a systematic review and meta-analysis. Eur Spine J 2020;29:2143-63.

10. Chiba D, Tsuda E, Wada K, et al. Lumbar spondylosis, lumbar spinal stenosis, knee pain, back muscle strength are associated with the locomotive syndrome: rural population study in Japan. J Orthop Sci 2016;21:366-72.

11. Lee SY, Kim TH, Oh JK, Lee SJ, Park MS. Lumbar stenosis: a recent update by review of literature. Asian Spine J 2015;9:818-28.

12. Weinstein JN, Tosteson TD, Lurie JD, et al. Surgical versus nonoperative treatment for lumbar spinal stenosis four-year results of the Spine Patient Outcomes Research Trial. Spine (Phila Pa 1976) 2010;35:1329-38.

13. Tomkins-Lane C, Melloh M, Lurie J, et al. ISSLS prize winner: consensus on the clinical diagnosis of lumbar spinal stenosis: results of an international Delphi study. Spine (Phila Pa 1976) 2016;41:1239-46.

14. Genevay S, Courvoisier DS, Konstantinou K, et al. Clinical classification criteria for neurogenic claudication caused by lumbar spinal stenosis: the NCLASS criteria. Spine J 2018;18:941-7.

15. De Graaf I, Prak A, Bierma-Zeinstra S, Thomas S, Peul W, Koes B. Diagnosis of lumbar spinal stenosis: a systematic review of the accuracy of diagnostic tests. Spine (Phila Pa 1976) 2006;31:1168-76.

16. Ishimoto Y, Yoshimura N, Muraki S, et al. Associations between radiographic lumbar spinal stenosis and clinical symptoms in the general population: the Wakayama Spine Study. Osteoarthritis Cartilage 2013;21:783-8.

17. Booth A, Clarke M, Dooley G, et al. PROSPERO at one year: an evaluation of its utility. Syst Rev 2013;2:4.

18. Spengler DM. Degenerative stenosis of the lumbar spine. J Bone Joint Surg Am 1987;69:305-8.

19. De Schepper EI, Overdevest GM, Suri P, et al. Diagnosis of lumbar spinal stenosis: an updated systematic review of the accuracy of diagnostic tests. Spine (Phila Pa 1976) 2013;38:E469-81.

20. Jenis LG, An HS. Spine update: lumbar foraminal stenosis. Spine (Phila Pa 1976) 2000;25:389-94.

21. Imai A, Kaneoka K, Okubo Y, et al. Trunk muscle activity during lumbar stabilization exercises on both a stable and unstable surface. J Orthop Sports Phys Ther 2010;40:369-75.

22. McNamara MJ, Barrett KG, Christie MJ, Spengler 
DM. Lumbar spinal stenosis and lower extremity arthroplasty. J Arthroplasty 1993;8:273-7.

23. Lee CY, Park BM, Kim TW, Lee SH. Clinical implication of mid-range dynamic instability in lumbar degenerative spondylolisthesis. Asian Spine J 2020;14:507-12.

24. Malfair D, Beall DP. Imaging the degenerative diseases of the lumbar spine. Magn Reson Imaging Clin N Am 2007;15:221-38.

25. Andreisek G, Imhof M, Wertli M, et al. A systematic review of semiquantitative and qualitative radiologic criteria for the diagnosis of lumbar spinal stenosis. AJR Am J Roentgenol 2013;201:W735-46.

26. Lee JW, Myung JS, Park KW, et al. Fluoroscopically guided caudal epidural steroid injection for management of degenerative lumbar spinal stenosis: short-term and long-term results. Skeletal Radiol 2010;39:691-9.

27. Norimoto M, Eguchi Y, Kanamoto H, et al. Diffusion tensor imaging of the spinal canal in quantitative assessment of patients with lumbar spinal canal stenosis. Asian Spine J 2020 Sep 3 [Epub]. https://doi. org/10.31616/asj.2020.0026.

28. Kent DL, Haynor DR, Larson EB, Deyo RA. Diagnosis of lumbar spinal stenosis in adults: a metaanalysis of the accuracy of CT, MR, and myelography. AJR Am J Roentgenol 1992;158:1135-44.

29. Oh Y, Shin DA, Kim DJ, et al. Effectiveness of and factors associated with balloon adhesiolysis in patients with lumbar post-laminectomy syndrome: a retrospective study. J Clin Med 2020;9:1144.

30. Qureshi F, Meena SC, Kumar V, Jain K, Chauhan R, Luthra A. Influence of epidural ropivacaine with or without dexmedetomidine on postoperative analgesia and patient satisfaction after thoraco-lumbar spine instrumentation: a randomized, comparative, and double-blind study. Asian Spine J 2020 Sep 3 [Epub]. https://doi.org/10.31616/asj.2020.0072.

31. Yukawa Y, Lenke LG, Tenhula J, Bridwell KH, Riew KD, Blanke K. A comprehensive study of patients with surgically treated lumbar spinal stenosis with neurogenic claudication. J Bone Joint Surg Am 2002;84:1954-9.

32. Kim HJ, Kim JH, Park YS, et al. Comparative study of the efficacy of limaprost and pregabalin as single agents and in combination for the treatment of lumbar spinal stenosis: a prospective, double-blind, randomized controlled non-inferiority trial. Spine J 2016;16:756-63.

33. Botwin K, Brown LA, Fishman M, Rao S. Fluoroscopically guided caudal epidural steroid injections in degenerative lumbar spine stenosis. Pain Physician 2007;10:547-58.

34. Manchikanti L, Cash KA, McManus CD, Pampati V, Abdi S. Preliminary results of a randomized, equivalence trial of fluoroscopic caudal epidural injections in managing chronic low back pain: part 4. spinal stenosis. Pain Physician 2008;11:833-48.

35. Thiengwittayaporn S, Koompong P, Khamrailert S, Wetpiriyakul P. Comparison of clinical outcomes of different rates of infusion in caudal epidural steroid injection: a randomized controlled trial. Asian Spine J 2020 Jul 24 [Epub]. https://doi.org/10.31616/ asj.2019.0380.

36. Park HJ, Kim SK, Lee SC, Kim W, Han S, Kang SS. Dural tears in percutaneous biportal endoscopic spine surgery: anatomical location and management. World Neurosurg 2020;136:e578-85.

37. Tram J, Srinivas S, Wali AR, Lewis CS, Pham MH. Decompression surgery versus interspinous devices for lumbar spinal stenosis: a systematic review of the literature. Asian Spine J 2020;14:526-42.

38. Lee BH, Yang JH, Kim HS, et al. Effect of sagittal balance on risk of falling after lateral lumbar interbody fusion surgery combined with posterior surgery. Yonsei Med J 2017;58:1177-85.

39. Lee BH, Park JO, Kim HS, et al. Spinal sagittal balance status affects postoperative actual falls and quality of life after decompression and fusion in-situ surgery in patients with lumbar spinal stenosis. Clin Neurol Neurosurg 2016;148:52-9.

40. Yang LH, Liu W, Li J, et al. Lumbar decompression and lumbar interbody fusion in the treatment of lumbar spinal stenosis: a systematic review and metaanalysis. Medicine (Baltimore) 2020;99:e20323.

41. Min WK, Kim JE, Choi DJ, Park EJ, Heo J. Clinical and radiological outcomes between biportal endoscopic decompression and microscopic decompression in lumbar spinal stenosis. J Orthop Sci 2020;25:371-8.

42. Nwosu KC, Khan SN, Cha TD. Lumbar spinal stenosis. In: Phillips FM, Lieberman IH, Polly DW Jr, Wang MY, editors. Minimally invasive spine surgery: surgical techniques and disease management. 2 nd ed. 
New York (NY): Springer; 2020. p. 417-28.

43. Kang T, Park SY, Park GW, Lee SH, Park JH, Suh SW. Biportal endoscopic discectomy for high- grade migrated lumbar disc herniation. J Neurosurg Spine 2020 May 15 [Epub]. https://doi. org/10.3171/2020.2.SPINE191452. 